\title{
A MOTHER-DAUGHTER MECHANISM OF MODE I CRACKS: SUPERSONIC CRACK MOTION ALONG INTERFACES OF DISSIMILAR MATERIALS
}

\author{
Markus J. Buehler ${ }^{1}$ and Huajian Gao \\ ${ }^{1}$ Max Planck Institute for Metals Research, Stuttgart, Germany
}

\begin{abstract}
In this paper we summarize recent progress in applying large-scale atomistic studies of crack dynamics along interfaces of dissimilar materials. We consider two linear-elastic material strips in which atoms interact with harmonic potentials, with a different spring constant in each layer leading to a soft and a stiff strip. The two strips are bound together with a weak potential whose bonds snap early upon a critical atomic separation. An initial crack serves as initiation point for the failure. We will focus on the maximum speed of mode I loaded cracks along such a biomaterial interface. Upon nucleation of the crack, it quickly approaches a velocity a few percent larger than the Rayleigh-wave speed of the soft material. After a critical time, we observe that a secondary crack is nucleated a few atomic spacings ahead of the crack. This secondary crack propagates at the Rayleigh-wave speed of the stiff material. If the elastic mismatch is sufficiently large, the secondary crack can be faster than the longitudinal wave speed of the soft material, thus propagating supersonically. Supersonic crack motion is clearly identified by two Mach cones in the soft material. This suggests that mother-daughter mechanisms, formerly only reported in mode II cracks in homogeneous materials, play an important role in interfacial mode I crack dynamics. The studies reported in this paper exemplify the usage of large-scale atomistic simulation to investigate the physics of fracture.
\end{abstract}

\section{INTRODUCTION}

Large-scale atomistic simulation is becoming an increasingly important tool to investigate fundamental aspects of dynamic crack propagation. Recent progress in this field include systematic atomistic-continuum studies of fracture [1,2], investigations of the role of hyperelasticity in dynamic fracture [3] and studies on the instability dynamics [4].

Here we extend existing atomistic models of dynamic fracture in homogeneous materials to study cracking along interfaces of dissimilar materials. Such investigations are motivated by both the scientific and technological relevance of this problem. From perspective of fundamental crack dynamics, the main question is which part of the bimaterial interface determines the crack limiting speed? The crack limiting speed, which is the Rayleigh-wave speed for mode I cracks, is associated with the elastic properties in the material. Since these change discontinuously across the interface, there is no unique definition of the wave speed. From the standpoint of technology, in many engineering applications, understanding of the properties of interfaces of dissimilar materials is crucial since they serve as potential failure sites in many composite materials. Yet another important field where interfaces between dissimilar materials play an important role is the dynamics of earthquakes.

Several theoretical studies were carried out on cracks in dissimilar media [5-7]. However, most of the early investigations focused on static cracks. One of the interesting features of the elastic interfacial crack problem is the characteristic oscillating stress singularity that was determined by Williams [8]. This theoretical finding is incompatible with real materials since the crack faces would penetrate each other at the crack tip. The stress intensity factor is complex-valued for interfacial cracks and it is generally difficult to define a crack nucleation criterion based on the Griffith condition [5]. 


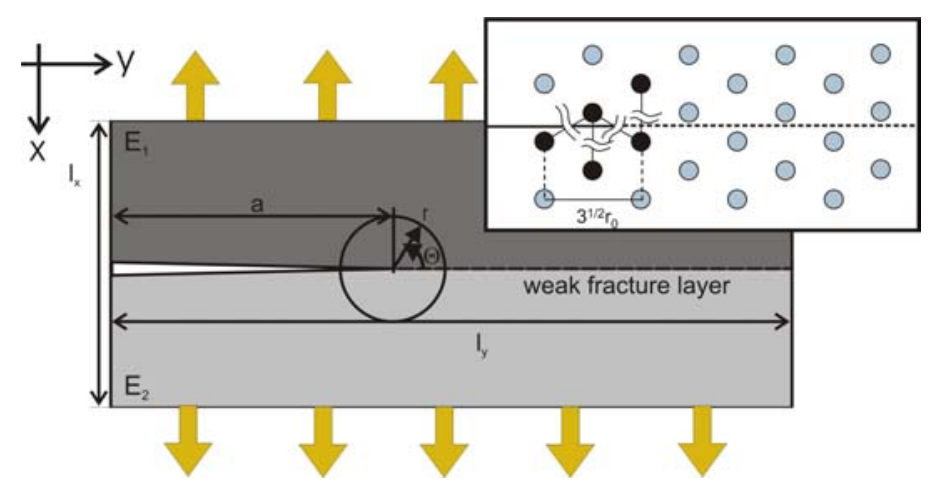

Figure 1:

Simulation geometry and lattice orientation for the studies of interfacial cracking. The figure shows the slab under mode I loading.

In recent years, the dynamics of moving cracks along dissimilar interfaces was increasingly in the focus of research. For instance, the asymptotic stress field near dynamic cracks at bimaterial interfaces was studied by Yang et al. (1991) and Liu et al. (1993) [9, 10]. The analysis discussed in [9] assumed steady-state crack propagation and provided the spatial structure of square-root singular stress field very close to the dynamic crack tip. The analysis led to definition of a complex dynamic stress intensity factor. Later, this analysis was refined relaxing the steady-state assumption and including higher order terms [10].

(a)

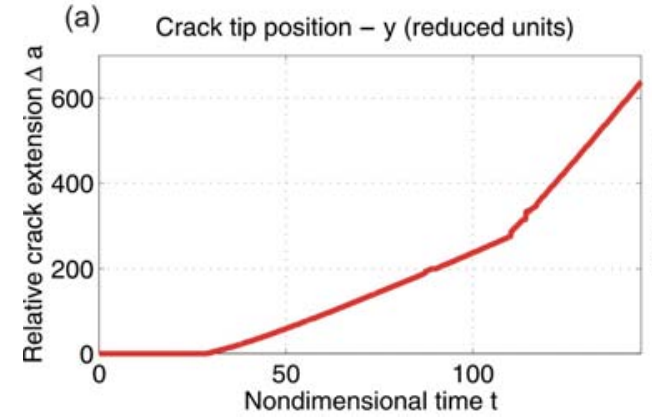

(b) Crack Tip Speed

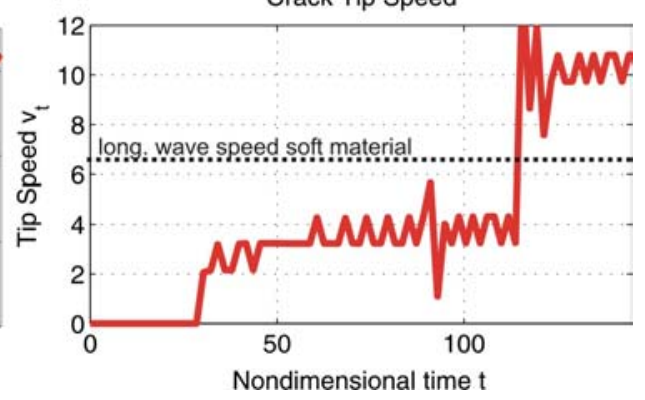

Figure 2: Crack tip history and crack velocity history for a mode I crack propagating at an interface with $\Xi=10$. Subplot (a) shows the crack tip history, and subplot (b) shows the crack tip velocity over time. A secondary daughter crack is born propagating at a supersonic speed with respect to the soft material layer.

There are also experimental results available on interfacial cracks, as for instance studies reported in [11-13] of cracks propagating along interfaces of PMMA and metals. In [11], the researchers focused on the development of a crack growth criterion along interfaces. They also compared the experimental results with theoretical predictions of the stress field near the crack tip. Crack speeds that exceeded that of the shear wave speed of the soft PMMA material were observed.

However, very few molecular-dynamics simulations of dynamic fracture along bimaterial interfaces have been reported. One rare example is recent molecular-dynamics simulations of mode II cracks along a weakly bonded interface of harmonic-anharmonic materials (material defined by a harmonic potential neighboring a material defined by a tethered LJ potential) [14]. 
For mode I cracks at interfaces, existing theory and experiment predicts that the limiting speed of mode I cracks at bimaterial interfaces can exceed the Rayleigh-wave speed of the soft material $[9,10]$. However, intersonic or supersonic crack propagation with respect to the soft material layer is not predicted by theory and has not been observed in experiment.

The most important research objective of our studies is the limiting speed of cracks: Using molecular-dynamics, can we determine what is the limiting speed of a crack along dissimilar materials? How fast can cracks propagate?

In an earlier study a mother-daughter-granddaughter mechanism was observed in mode II cracks, by which the crack finally approached a velocity faster than the longitudinal wave speed of one of the layers [14]. In this setup, however, one of the half spaces was modeled by harmonic interactions, and the other was modeled by a tethered Lennard-Jones potential (that is known to yield a material behavior stiffening with strain). Although this setup constitutes an interface of different materials, the wave speeds associated with each half space could not be clearly defined since one of the materials was hyperelastic. To obtain a cleaner model of cracks at interfaces, we propose to study two half spaces with harmonic interatomic potentials, but with different spring constants $k_{2}<k_{1}$. The ratio $\Xi=k_{2} / k_{1}$ measures the elastic mismatch of the two materials, and the wave speeds are thus different by a factor $\sqrt{\Xi}$.

The outline is as follows. This paper focuses on atomistic studies of mode I cracks along interfaces. We show that under sufficiently large loading, the crack approaches the Rayleigh wave speed of the stiffer of the two materials via a mother-daughter mechanism. A detailed atomic-scale analysis of the nucleation mechanism is included that sheds some light on the nucleation mechanism, suggesting that a peak in shear stress ahead of the crack causes nucleation of the secondary crack.
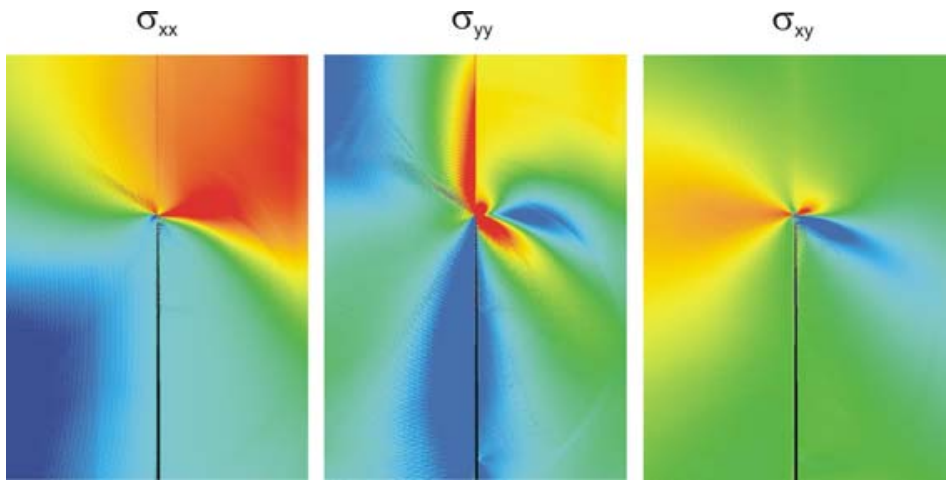

Figure 3: Stress fields $\sigma_{x x}, \sigma_{y y}$ and $\sigma_{x y}$ for a crack at an interface with elastic mismatch $\Xi=10$ before the secondary crack is nucleated. The red color corresponds to large stresses, and the blue color to small stresses.

\section{MODE I CRACKS AT BIMATERIAL INTERFACES}

In the simulations, the left part of the slab corresponds to the stiff part, while the right part has lower Young's modulus and is thus soft. We consider the case when the elastic mismatch is $\Xi=10$. For comparison, the elastic mismatch as between PMMA and aluminum is about 15 . Figure 2 (a) shows the crack tip history, and Figure 2 (b) shows the crack tip velocity over time. The crack nucleates at time $t \approx 35$, and quickly approaches the Rayleigh speed of the soft material $v \rightarrow c_{r, 0} \approx 3.4$. As loading is increased, the crack speed increases slightly and becomes super-

Rayleigh. We observe a large, discontinuous jump in the crack velocity at $t \approx 110$, when a secondary crack is nucleated that quickly approaches the Rayleigh speed of the stiff material 
$v \rightarrow c_{r, 1} \approx 10.8>c_{l, 0} \approx 6.36$. The secondary crack is nucleated approximately at a distance

$\Delta a \approx 11$ ahead of the mother crack and rapidly propagates with Mach 1.7 through the material! Nucleation of secondary cracks under mode I loading is only found under high-strain rate loading (here we used $\dot{\varepsilon}_{x x}=0.000,05$ ). If the strain rate is too low, the crack moves at a super-Rayleigh speed until the solid has separated, without nucleation of secondary cracking. The mechanism of nucleation of a secondary crack is reminiscent of the mother-daughter mechanism of mode II cracks, a phenomenon commonly observed in cracks under shear loading.

The result suggests that at a bimaterial interface, mode I cracks under very large loading can propagate with the Rayleigh speed of the stiffer materials, and cracks can reach speeds beyond the fastest wave speeds in the soft material. This observation is surprising and has not been reported in experiment so far [13], nor has it been predicted by theory. In experimental studies of mode I cracks along interfaces, the crack slightly exceeds the Rayleigh speed of the soft material, but it is never observed to attain supersonic velocities.

As the next step, we take a closer look at the deformation fields before and after nucleation of the secondary crack. Figure 3 shows the stress field before the secondary crack is nucleated. At the time the snapshots are taken, the crack propagates at a (slightly) super-Rayleigh speed through the material. Since crack motion is subsonic, no shock front is established. Figure 4 shows the stress field and the particle velocity field after nucleation of the secondary crack. The secondary crack propagates supersonically through the material and the Mach cones in the right half space (soft material) are clearly visible. Figure 5 shows the potential energy field for a crack after the secondary, supersonic crack is nucleated. The two mach cones can clearly be observed. The mother-daughter mechanism in mode I cracks at interfaces is also observed for elastic mismatch $\Xi=2, \Xi=5, \Xi=7$ and (note that not in all cases crack motion is supersonic with respect to the soft material since the Rayleigh-wave speed of the stiff material is smaller than the longitudinal wave speed of the soft material).

What is governing nucleation of the secondary crack? MD simulations can be a helpful approach in investigating the atomistic details of the mechanism of nucleation of secondary cracks. In Figure 6, we plot the shear stress field $\sigma_{x y}$ for several instants in time during nucleation of the secondary crack (note we chose quite small time intervals between the snapshots). Atoms with the energy of a free surface are colored blue and highlighted by larger radius spheres. The plot suggests that at a maximum peak of the shear stress ahead of the crack tip leads to breaking of atomic bonds by shearing and creation of surface. After the secondary crack is nucleated, it coalesces with the mother crack and moves supersonically through the material.
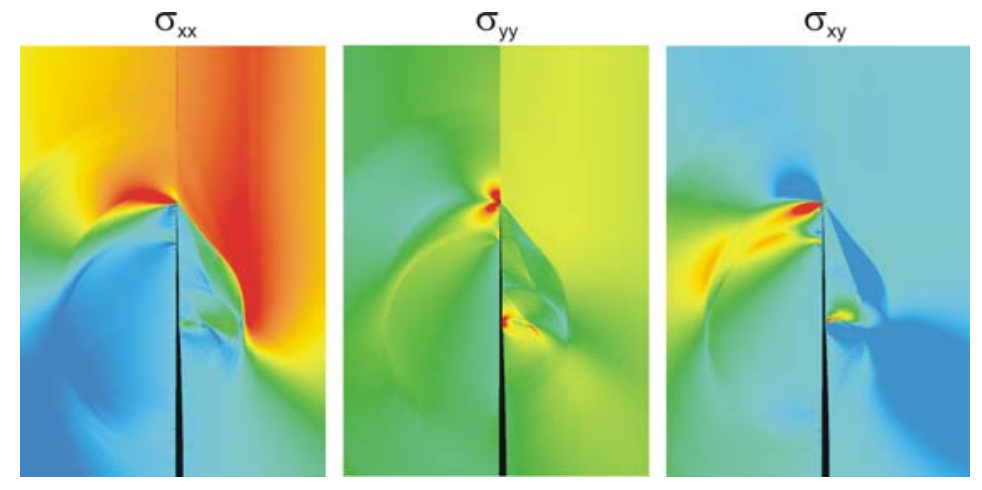

Figure 4: Stress fields $\sigma_{x x}, \sigma_{y y}$ and $\sigma_{x y}$ for a crack at an interface with elastic mismatch $\Xi=10$ after the secondary crack is nucleated. In all stress fields, the two Mach cones in the soft material are seen. 


\section{DISCUSSION AND CONCLUSIONS}

The studies reported in this section show that cracks at interfaces show a very different dynamics than cracks in homogeneous materials. At the interface, the limiting crack speed is not well-defined since the wave velocities change discontinuously across the interface. We find that mode I cracks can propagate supersonically with respect to the wave speeds in the soft material. We observe that the limiting speed of cracks at bimaterial interfaces is the Rayleigh-wave speed of the stiff material.

The observation of a mother-daughter mechanism in mode I cracks is a new phenomenon in dynamic fracture that was not reported in the literature so far. It is also in contrast to published experimental results [13], where cracks are found to be limited by super-Rayleigh speeds.

Preliminary continuum mechanics analysis stimulated by our atomistic simulation results provides theoretical evidence that this dynamical phenomena is indeed possible, since the analysis revealed that the energy release rate is positive for crack motion at the Rayleigh-speed of the stiff material (and supersonic crack speed with respect to the soft material) [15].

The elastic fields establish very differently from homogeneous materials. If crack propagation is supersonic with respect one of the half spaces, multiple shock fronts are observed as shown in Figure 4 . The elastic fields in the left and right half are asymmetric, which matches the predictions by continuum mechanics theories qualitatively [10]. A more detailed comparison of continuum theory and MD simulation is left to future work.

A preliminary analysis of the mechanism of nucleation of secondary cracks was shown in Figure 6. The results suggest that a peak in shear stress ahead of the crack causes nucleation of a secondary crack that moves at the Rayleigh-wave speed of the stiff material. Continuum mechanics analysis has shown that crack propagation speed at the Rayleigh-wave speed of the stiff material is in principle possible [15], thus supporting the discoveries reported in this paper.

The results suggest that atomistic simulations are a feasible approach to study the dynamics of cracks at interfaces. The present study exemplifies the usage of large-scale atomistic simulation to investigate the physics of fracture. A similar approach could be used in studying cracks under geometric confinement, crack tip instabilities or the effect of hyperelasticity on crack dynamics.

\section{ACKOWLEDGEMENTS}

Most of the simulations were carried out in the Garching Supercomputer Center of the Max Planck Society, we gratefully acknowledge their support.

$$
\mathrm{e}_{\mathrm{pot}}
$$

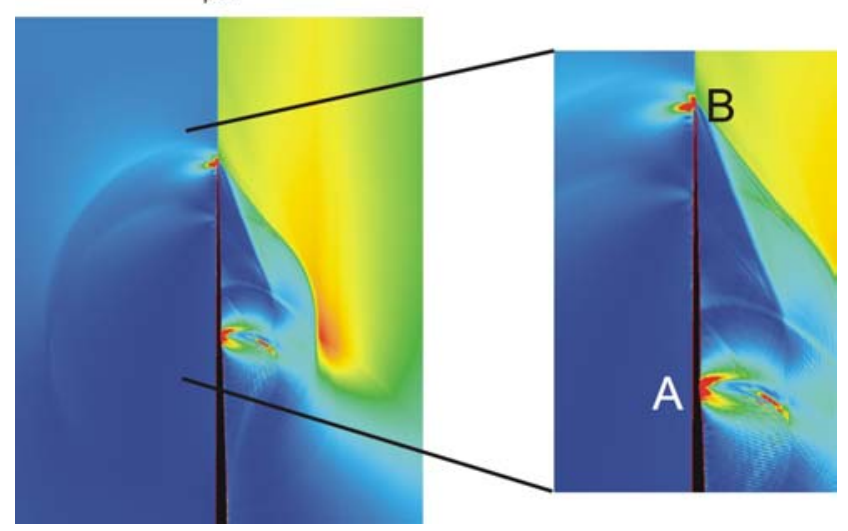

Figure 5: The plot shows the potential energy field for a crack at an interface with elastic mismatch $\Xi=10$. Two Mach cones in the soft solid can clearly be observed. Also, the mother and daughter crack can be seen. In the blow-up on the right, the mother $(\mathrm{A})$ and daughter crack (B) are marked. The mother crack is represented by a surface wave in the soft material. 

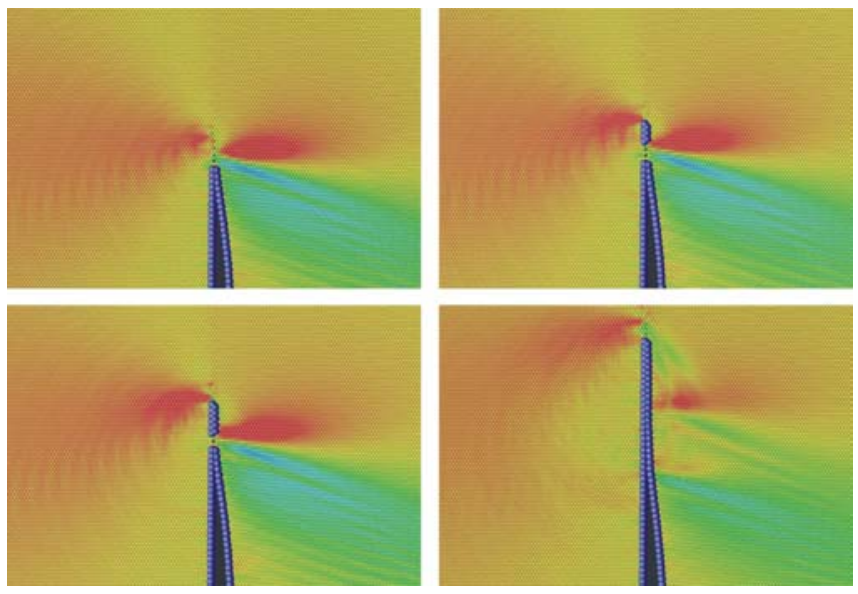

\section{REFERENCES}

1. Gao, H.J., Huang, Y., Abraham, F.F. Continuum and Atomistic Studies of Intersonic Crack Propagation, J. Mech. Phys. Solids 49, 2113-2132 (2001).

2. M.J. Buehler, F.F. Abraham, H. Gao, Hyperelasticity Governs Dynamic Fracture at a Critical Length Scale, Nature 49, 441-446 (2003)

3. M.J. Buehler, H. Gao, Y. Huang, Continuum and Atomistic Studies of Suddenly Stopping Supersonic Cracks, Computational Materials Science 28, 385-408 (2003).

4. Abraham, F.F., Brodbeck, D., Rudge, Instability Dynamics of Fracture: A Computer Simulation Investigation W.E., Xu, X. Phys. Rev. Lett. 73, 272-275 (1994).

5. Rice, J.R., Sih, G.C. Plane Problems of Cracks in Dissimilar Media. Trans. of the ASME 32(2), 418-423 (1965)

6. England, A.H. A Crack Between Dissimilar Media. J. Appl. Mech. 32, 400-402 (1965)

7. Rice, J.R. Elastic fracture mechanics concepts for interfacial cracks. Trans. of the ASME 55(1), 98-103 (1988)

8. Williams, M.L. The stresses around a fault or crack in dissimilar media. Bull. Seismol. Soc. America 49, 199-204 (1959).

9. Yang, W., Suo, Z., Shih, C.F. Mechanics of dynamic debonding, Proc. Roy. Soc. Lond. A 433, 679-697 (1991).

10. Liu, C., Lambros, J., Rosakis, A.J. Highly transient elastodynamic crack growth in a bimaterial interface: higher order asymptotic analysis and experiments. J. Mech. Phys. Solids 41, 1887-1954 (1993).

11. Lambros, J., Rosakis, A.J. Development of a dynamic decohesion criterion for subsonic fracture of the interface between two dissimilar materials. Proc. Roy. Soc. Lond. A 41, 711736 (1995)

12. Rosakis, A.J. Intersonic crack propagation in bimaterial systems. J. Mech. Phys. Solids 6(10), 1789-1813 (1998).

13. Rosakis, A.J. Intersonic shear cracks and fault ruptures. Adv. Phys. 51(4), 1189-1257 (2002).

14. Abraham F.F et al. Simulating Materials Failure by using up to one billion atoms: Brittle Fracture. Proc. Nat. Acad. Sci. 99, 5788-5792 (2002).

15. Chen, S. Personal communication. 